\title{
ONLINE IDENTITY: \\ CONSTRUCTING INTERPERSONAL TRUST AND OPENNESS THROUGH PARTICIPATING IN HOSPITALITY SOCIAL NETWORKS
}

\author{
Alexander Ronzhyn \\ a.ronzhyn@deusto.es \\ University of Deusto (Bilbao, Spain)
}

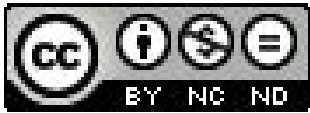

\begin{abstract}
The present article describes the results of research on online identity construction during the participation in the hospitality social networks. Specifically the user references are analysed to understand patterns that form the image of a member. CouchSurfing service (couchsurfing.org) allows users to leave short texts where the experience of hosting/being hosted by a CS member is described, is an evaluation of the CS members of each other's personal traits, skills and common experience. Therefore references can become a good instrument for portraying a CouchSurfing member and understanding his or her particular traits. References form an important part of a user's virtual identity in the network. Using a sample of references of Spanish CouchSurfing users, the research established main characteristics of the references, which are the openness, readiness to share ideas and experiences and trustworthiness. These concepts illustrate the typical traits associated with a user of the network and also shed light on the activities common during offline CS meetings
\end{abstract}

Key words: identity construction, online identity hospitality social networks, CouchSurfing

\section{INTRODUCTION}

Hospitality social networks are the Internet social network services (SNS) used to arrange free accommodation during travel. These networks developed from the offline hospitality exchange directories and clubs. These early clubs allowed their members to access a special directory (essentially a yearly book) with contact details of other members, in order to arrange stay during travel. Early hospitality exchange networks did attract attention - US president Jimmy Carter was a founder of one such network: Friendship Force International, although they never became ubiquitous and popular enough to substantially affect the travel routines of many people. Everything changed after a rapid ascent of the Internet as a communication technology: communication had never been so effortless and everything that depended on the speed of human interactions naturally began to migrate to the web. Internet hospitality exchange networks were one of the first to benefit from social media boom. In 2004, a year before Facebook, couchsurfing.org became the first hospitality service to exhibit all three defining properties of an SNS. It had user-created profiles, 
visible connections between users and a way to interact through these connections. In the following years CouchSurfing steadily grew in popularity, attracting large amounts of media attention in the process. It has reached 4000000 users in 2012 and has become a truly global phenomenon (by comparison, the largest still existing offline hospitality exchange, the Friendship Force International has only 18000 members). "Couch surfing" itself became a euphemism for the activity promoted by the website. So far online hospitality exchange services have not attracted much academic attention. Most notable works include study of online familiarity by Paula Bialski (2012), management of trust within hospitality SNS by Lada A. Adamic, Debra Lauterbach and Chun-Yuen Te (2011), and the research on interpersonal trust in CouchSurfing by Le Dieu Tran (2009).

While CouchSurfing is also an SNS like Facebook, MySpace or Twitter, it should be approached separately as it has several important differences from more conventional networks. Firstly and the most significant is the nature of interactions between users: while in Facebook most of the connections are formed between people who had already known each other offline, the so-called weak ties, hospitality SNS in their essence are designed to encourage and facilitate the communication between strangers, people who had never met before. From this point of view hospitality SNS are closer to traditional dating sites than to the networks like Facebook. The second important difference also evokes a similar connotation: the final goal of the interaction between users in the hospitality network is meeting in the real life. MySpace, Facebook, Google+ are all designed to provide immersive online experience for the Internet user, so the user can maintain its egocentric network, share content and experiences without any need for offline communication. Contrary to that, CouchSurfing is designed in a way to arrange, prepare and simplify the transaction from online to offline communication. In a sense, online experience in hospitality SNS is secondary to the offline interaction. This makes networks like CouchSurfing a particularly interesting and a tempting subject for research into how online and offline components relate and interplay in the lives of Internet users.

\section{IDENTITY CONSTRUCTION ONLINE}

One of the distinctly interesting discussions within the Internet research is centred on the question of how Internet users construct their identity. Online identity construction is one of the most interesting and research intensive focuses of Internet studies, although it is worth noting than most of the works dealing with identity construction on the Internet study the identities in the conditions of anonymity. Only with the rise of Facebook, studies of the identity constructions in the real-name communities began to appear (Zhao, Grasmuck, \& Martin, 2008). Just like Facebook, CouchSurfing is not an anonymous community. Contrary to many other internet communities hospitality exchange networks demand the reality of identity information and addresses. This is done through verification processes. On CouchSurfing verification is a routine that demands the authenticity of the name and address, established through a "verification payment" with a card with the name and billing address corresponding to the one indicated on the website. Because of its nature and 
inevitability of offline user interactions, CouchSurfing (and other hospitality SNS as well) practically require the authenticity not only of the members' names, but of also of addresses and photos. Such de-anonymisation is both directly encouraged by the system (through the system of verification) and conditioned by the inner ethics of member interaction. As all the interaction in the hospitality networks is based on trust, photos are often required by hosts to form an image of a future visitor before letting her or him into the home. Related research dealing with real-name networks includes works on identity construction in Facebook by Shanyang Zhao, Sherri Grasmuck and Jason Martin (2008), Soraya Mehdizadeh (2010), Katie Ellis (2010), identity construction in Twitter was discussed by Dawn R. Giplin (2011).

As most of the user-to-user communication on CouchSurfing is meant to culminate in an offline meeting, identity construction in the hospitality SNS ideally should serve two functions. Firstly, identity should be trustworthy enough to serve its purpose, either being accepted as a guest by the hosting user, or be visited by the guest. Secondly, it should be the representation of a member. CouchSurfing users represent themselves primarily through their profiles. Profile information is what forms a user's identity in the network. Couchsurfing profiles are the subject of the present research and the references which constitute a part of a profile are what was analysed during the course of the study. Similar to the profile pages on any other SNS, CouchSurfing profiles contain both user- and system-generated information. User-generated information is used for self-presentation and the direct construction of identity. System-generated data is the reflection of the user's activity in the network; it includes lists of "vouch" and "friend" connections, references and statistical information. User-generated information is the interesting part for the purposes of the present paper - as it depends solely on a user and basically forms his or her identity in the network. In their research Paula Bialski and Dominic Batorski outlined three stages of the user experience in the CouchSurfing network, when the trust emerges. Pre-selection of users, website navigation and offline contact are the three dimensions of CouchSurfing usage that form a familiar environment and allow the trust between the community members. The second stage: website navigation is actually the creation and interaction through and with the help of the user profiles and it is arguably the most significant one (Bialski, \& Batorski, 2010, p. 202). Profiles are the sources of trust in users and they are what other members evaluate when they judge whether a profile owner may be trusted. The profile in CouchSurfing is structured in such a way that when a user creates one, he or she answers a specific set of questions: "What is your personal philosophy?", "Why do you live your life the way you do?", "most amazing thing you've done?" and others. Whilst the way these questions are laid out creates a more trusting environment by making profiles more similar and thus familiar, it is the content that is evaluated by the other users to form an image about other hospitality network members. The apparent structure of the profiles also allows more refined and precise comparison of different users' profiles as this structure undoubtedly somewhat limits the member's activity of expression, making the profiles more similar to open-ended survey responds than to the free compositions on the vaguely identified topic.

References are part of a user's profile created as a result of a user-to-user interaction. Basically a reference is a usually a short comment left by the host and guest after 
the visit took place. There are no specific restrictions established by the system apart from the maximum number of words which is 1000 . Typically though a reference is 40-150 words long and resembles a "thank you" note describing the time spent together and recommendation for other members concerning a particular host/guest. References may be neutral, negative or positive, but in reality the distribution is very uneven: roughly $90 \%$ of all references are positive (Adamic, Lauterbach, \& Te, 2011, p. 7). The references are meant to be reciprocal, yet sometimes a reference is left only by one party. One possible reason for declining to leave a reference may be the unwillingness to leave a negative one.

\section{RESEARCH ON TRUST IN HOSPITALITY NETWORKS}

One important concept analysed in this paper is trust and its representation in the user profiles as a part of their Internet social identity. For the purposes of the present paper trust is defined according to the Lynne Zucker's definition as "an expectation or one's belief that the other party will behave in a dependable, ethical, and socially appropriate manner" (Zucker, 1986) and applied towards the unknown other social network users, essentially strangers. In this sense it is generalized social trust (Delhey, \& Newton, 2004) that is being exercised by members of the social networks towards each other, when they have not engaged in any interaction, so that the trust might be affected by some other (personal) reasons. The existing research of trust on the online social networks followed two paths. The first direction is aimed at computing and objectivizing trust for the purposes of the design improvement of existing and future online services. In this case trust is closely connected to privacy and safety on the Internet. The final goal of this research is to provide intending users with reliable and trustworthy information, making the Internet experience safer and more reliable (see Smith, 2009 and Furht, 2010). Still, at present, managing trust online remains the area controlled primarily by the users, who may grant or restrict access to their information, using the tools provided by the services they use. The second direction of the trust research online deals exactly with the way people control their privacy and manage trust during the interactions on the Internet (Bhulyan, Josang, \& Xu, 2010). This research of practical aspects of managing trust in the SNS concentrate on sociological and behavioural aspects of participation in social networks, privacy issues and the way the egocentric social networks are constructed by SNS members (see (Dwyer, Hiltz, \& Passerini, 2007) (Trepte, \& Reinecke, 2011)). The existing research is however limited to a number of most popular SNS. Facebook as the most widespread social network is the most common subject of analysis (see Johnson, Lavesson, Zhao, \& Wu, 2011; Dwyer, Hiltz, \& Passerini, 2007).

Trust is connected with familiarity. In the context of the Internet this suggests that more familiar and understandable services appear more trustful. And in the hospitality exchange SNS more familiar users are more likely to interact or connect with each other (Bialski, \& Batorski, 2010, p. 190). Indeed research confirmed that more similar network members are significantly more likely to interact than very different ones. It was also established that trust is formed through the interaction with user profiles, and information contained in the profiles was the main factor that affected 
the person's (dis-)trust towards other network member (Bialski, \& Batorski, 2010, p. 180). Profiles are the main focal point of the present research for the same reason: they are the main source of user information in the network and simultaneously the main tool used for identity construction. Another research on familiarity of trust conducted by Jie Zhang (2004, p. 23) suggested the possibility of measuring online familiarity through calculating several quantitative factors. The factors described by the author suggest that measuring familiarity (and thus trust related to familiarity) is advisable while analysing controlling for the amount of previous experience with the service. For that reason the sample used for the present work's analysis consists of users with similar backgrounds in using the CouchSurfing service.

\section{METHODOLOGY OF THE STUDY}

Investigating the ethnography of Web, we use a plural paradigm under the general umbrella of Web Content Analysis. This complex method was explained and described in the research by Susan Herring in 2010 and is defined as a broader construal of web content analysis, one that goes beyond traditional content analysis, drawing on methods from other disciplines (in particular linguistics and sociology) (Herring, 2010). Web content analysis in this case should be understood as analysis of web content using various (traditional and non-traditional) techniques. Integrating different methods into the analysis of the users profile enables us to deal properly with multimodal structure of a hospitality social network and cover a broad range of content.

Plural, broad or expanded Web Content Analysis paradigm involves methods of discourse analysis and social network analysis that are operationalized and implemented within a general content analytic framework, addressing characteristic features of the web. Besides, the methods of traditional content analysis adapted to the researching the blogs are applied. Blogs resemble the profiles on couchsurfing. org because of their structure. Profiles the same as blogs don't consist of texts only. Typically they are structured in various parts and the location and formatting of the text can be significant, not only the direct meanings of words. In case of analysis of profiles we can take into account the links, announcements, participation in events, membership of belonging to special groups etc. These factors can influence the interpretation of the results. Thus analysis units, similarly to other researches of blogs 7 , include information blocks about the user, images, description of the host's apartment and references. The expanded methodological approach to content analysis considers content not just as thematic meanings present in text but interprets content as various types of information "contained" in the user profiles on CouchSurfing website, including features, links, and exchanges, all of which can communicate meaning. However in this particular research we analyse only one structural unit of a profile: references. A broader analysis of multimodal and multifunctional web content of hospitality social networks (e.g. images, connections, references, links etc.) is still to be done. References are the short texts (usually up to 150 words) where the users describe and evaluate the experience of hosting or being

Such features of blogs were analyzed by Herring, Scheidt, et al. $(2004,2005,2006)$ using a classical CA approach. 
hosted by the particular CS user. We consider content framed within the references left on users' profiles on CouchSurfing as socio-cultural text. Therefore the elements of discourse analysis are applied to the profiles. Describing and exploring the texts, we are trying to uncover the "underlying meaning and significance" (Herring, 2010) of the texts. References normally don't include any other content except for the text. We also don't take into account whether the reference is positive, negative or neutral (the ranking system is integrated in the web). Thus analysing the references we apply traditional content analysis and discourse analysis. 288 references/ 144 pairs (usually both members of CS leave the references after the meeting). The criteria for choosing these particular 288 references is the geographical location of the member: references analysed were left by/for the members from the Basque country in Spain (primarily Vitoria and Donostia regions). When analysing the texts the fact that most of them were written by not-native-speakers was also taken into account. The rationale for choosing the language-focused approach combined with traditional content analysis is that in an online environment the human behaviour is carried out mostly through linguistic means (Herring, 2010). And in this particular case the texts become the instrument of an online identity construction.

The aim of the research is to analyse how the categories of trust and openness are expressed though the references left by/for the members of couchsurfing.org. The data for analysis is 288 references / 144 pairs by/for the experienced members from Basque country (primarily Vitoria and Donostia regions), collected in December 2012. We defined experienced member as the one having received at least ten references and active at least once in the last 6 months. For the present sample only positive references were collected as only 2 negative and one neutral reference were found while analysing intended profiles and no significant conclusions could be made judging such a small number of references. Carefully reading the references we took into account not only the precise phrases and words used by the users, but also analysed the word choice and the general style of the text according to the categories chosen.

\section{RESULTS: INTERPERSONAL TRUST AND OPENNESS}

Interpersonal trust. The unit of reference itself is a very important element in the structure of a CoushSurfing profile. Normally the CS members tend to ignore the requests without references or at least handle them cautiously. However reference is not a necessary requirement to participate in the community, it is a basic element that helps to create the atmosphere of mutual trust within the social network. Most of the references left on CS are positive, though negative ones also exist. If in other social networks like Facebook or MySpace the user constructs his or her identity as he wants others to see him/her, CouchSurfers are allowed to construct their online identity only partially. The rest of the work is done by other members of a community through the references they leave. Reference-based identity and profile identity constructed by the CS user him/herself can differ. For instance, one of the references states: "No matter how scary his profile seemed at the very beginning (to me at least; ;)), $R$. is a great and entertaining person and I really enjoyed his company and jokes" [sic]. User $\mathrm{R}$. wanted others to see him as a gloomy person, but references left by other users, 
who stayed at his place construct another identity of R., showing up his sense of humour, etc. Reference is a fundamental brick in building the trustworthy profile, because it is the only element of a profile (apart from statistics) that the member him/herself cannot influence. The simple tool that allows the CS members to evaluate and describe their hosting or surfing experience helps to create a social network with an "atmosphere of trust", described in one of the references. The CS members however rarely refer directly to the categories of trust in their references, preferring to describe the shared activities and personal traits of their host or a guest. It may be also suggested that writing that "you can trust him/her" is likely to be interpreted as if the member had doubts in trusting the particular user before meeting him or her personally. From 288 references only nine people write directly that a person is trustworthy, one of them thanks for trusting. The other way to express the trustworthiness of a member is to use language constructions addressing the potential hosts/ surfers of a member. The word "you" contain 140 references and 30 of them contain the structures "you can/ you have to/ you should". For instance: "If you host them", "with whom you can have a great time, you can talk about everything with him", "I highly recommend that you host her, you should definitely get in contact with him", "definitely a person that you have to meet", etc. 13 references contained direct recommendations to host or surf a couch of the referenced member, using the verb "recommend". References, being an instrument for making people trust each other in a virtual community contain direct messages of trust. Describing the shared experiences is also aimed at making other people interested in a particular user. In other words references are the disclaimer of trust within couchsurfing.org.

Openness. The single important mantra and the network-defining notion on CouchSurfing is openness. It is both arguably the most popular self-defining characteristic in the member profiles and at the same time the most wide-spread attribute of a good couchsurfer as described in the references. Partially because the openness to new experience and other people is the cornerstone of CouchSurfing sharing philosophy and is seen as a definite virtue for a member, and partially because "open" is a trait neutral enough for one to call him/herself so and not appear boastful. Almost a quarter of all references characterized a person on the receiving end as open or open-minded either directly or implying that by referring to a particular activity or attitude. Such references included mention of the willingness to discuss one's culture ("She shared her culture with us", "I would greatly recommend the experience if you are truly interested in learning more about the local culture", "Thank you for your kind explanations about different cultures"), member's knowledge of other culture ("he was speaking Euskera like a native", "he explained local traditions to us"), or indirectly, referring to the ability to listen and respect others. The willingness to share or exchange something is also expressed fairly often. 14 references contain the keywords "share/ exchange", where stories, experiences, cultures, conversations, love for life, trip are shared. Out of 288 analysed references in total 62 references contained a mention of the openness of the referenced party, making "openness" the single most frequent positive characteristic in the sample.

Ability and desire to learn as well as the corresponding activity of tutoring is another recurring theme. As a result 31 references relate to learning or teaching. The members 
are both positively described as people eager to learn and people able to teach something (ready to learn new things, learns amazingly fast, would like to continue learning, teach, he has taught me more about Norway than I ever knew before). CouchSurfing is very often a transnational activity that's why it often becomes an instrument for learning new languages (especially in Europe where the concentration of diverse nations and languages is much higher than on the other continents). Besides that, considerable numbers of couchsurfers ( $28 \%$ of hosts in the sample) who practice couchsurfing in the Basque country are foreigners coming from a variety of different states. All this frequently makes couchsurfing a multilingual experience, and although in most cases users have English as a common language, the actual level of the language differs and more than that, the relation between different languages is a popular topic for CS conversations.

Fig. 1. The most popular words found in the references. This tag cloud pretty well sums up the content of a typical reference left on CouchSurfing

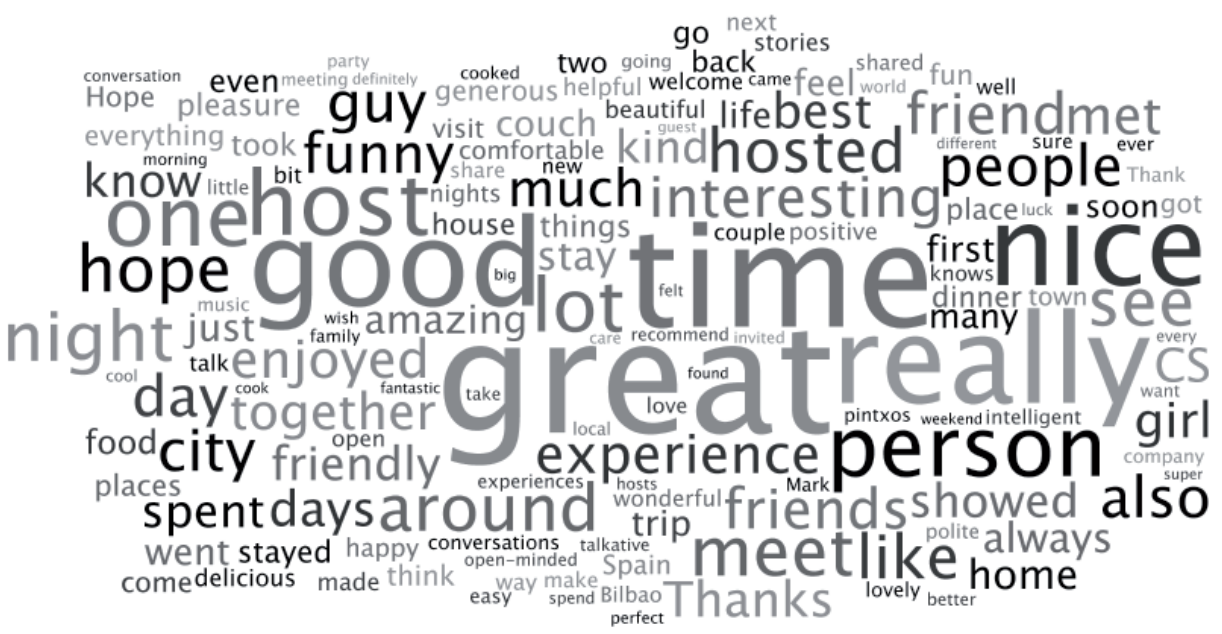

Source: Created by the author

Significant numbers of keywords relate to the positive mental and social characteristics of the referenced members. Such attributes are usually listed in succession, praising a successful couchsurfing experience ("He is just so intelligent, interesting, easygoing and generous", "They are sensitive, cultivated, respectful, humble, funny, laid-back, easygoing, curious"). Judging from how often they can be seen in references, such lists are more the signs of politeness bound by etiquette than objective descriptions of the person. It is worth noting though, that these attributes tend to levitate towards social aptness (the most common attributes being interesting (42), friendly (36), generous (21), positive (20) and easy-going (15), helpful (39) all relating mostly to the extroverted type of a person. Such clear prioritization depicts the SNS's focus on sharing and participative behaviour. In total more than $92 \%$ of all sample references contain positive descriptive attributes, of which $76 \%$ are those related to communicated skills. 
Another relatively common theme in the references is CouchSurfing itself. 5.2\% of all references contained mention of CouchSurfing values, mission or spirit. Usually such mentions were coupled with positive valuation of someone's hospitality, respectfulness or willingness to help. Respect for CouchSurfing values is also connected to trust ("I think she fits perfectly to CS mission. I would trust her with my life").

\section{CONCLUSIONS AND FUTURE RESEARCH}

The analysis of the references sample of Spanish CouchSurfing members revealed the tendency of users to concentrate on social traits when writing a reference for the other couchsurfing.org member. Among these traits openness and desire to share (experiences, own culture, ideas) are the most wide-spread. Trust, respectfulness and the respect of the implicit CS mission and values are also a popular topics included in the references. Common activities are also an attribute of successful couchsurfing experiences as they were mentioned in a fifth of all references. As a result of this research an outline of a typical positive reference can be created, mentioning the most valuable and important traits for a couchsurfer as seen by other CouchSurfing members: interesting, open-minded, generous, positive, trustful, easy-going, helpful and willing to share.

The content-analysis of the references may also help to reveal the most common shared activities of the members during CouchSurfing. Even cursory examination reveals plenty of information related to the activities undertaken by the members. $20.2 \%$ of examined references contain mention of a specific shared activity: $5.9 \%$ (17) speak of "town tour", "excursion" or "guide through town", 10\% (29) mention shared cooking and $7.2 \%$ (21) have mention of visiting bars or restaurants. Another frequent activity is language tutoring. User references may prove to be a valuable and objective view on the way CouchSurfing members communicate offline. Such indirect observation may be useful when studying this aspect of hospitality exchange, though undoubtedly further sophistication is needed to usefully apply the analysis to the references data sample and a larger sample is also advisable.

Further development of the analysis may lead to an increase of the analysed sample size, application of the approach to a wider geographical zone and inclusion into the analysis not only the references but also "friends comments" - short descriptions that accompany the friend-connection on CouchSurfing. Besides, it would be interesting to research the question of identity construction comprehensively, comparing and examining profile information generated both by other users and by the profile owner. Analysing a bigger sample may allow the establishing of patterns and concepts that were missed due to the limitation of the present work.

\section{REFERENCES}

Adamic, L., Lauterbach, D., \& Te, C.-Y. (2011). Rating Friends Without Making Enemies. Fifth International AAAI Conference on Weblogs and Social Media. Retrieved March 27, 2012, Retrieved from: http:/ / www.aaai.org/ocs/index.php/ICWSM/ICWSM11/paper/view/2837/3280

Bhulyan, T., Josang, A., \& Xu, Y. (2010). Managing Trust in Online Social Networks. In: B. Furht, Handbook of Social Network Technologies and Applications (pp. 471-496). Berlin: Springer-Verlag. 
Bialski, P. (2012). Becoming Intimately Mobile (Vol. 2). Warsaw: Warsaw Studies in Culture and Society.

Bialski, P., \& Batorski, D. (2010). From Online Familiarity to Offline Trust: How a Virtual Community Creates Familiarity and Trust between Strangers. In: P. Zaphiris, \& C. S. Ang, Social Computing and Virtual Communities (pp. 179-205). Boca Raton, USA: Taylor and Francis Group.

Delhey, J., \& Newton, K. (2004). Social Trust: Global Pattern or Nordic Exceptionalism? Berlin: Wissenschaftszentrum Berlin für Sozialforschung (WZB).

Dwyer, C., Hiltz, S., \& Passerini, K. (2007). Trust and Privacy Concern Within Social Networking Sites: A Comparison of Facebook and MySpace. AMCIS 2007 Proceedings. Paper 339. Retrieved from http:// aisel.aisnet.org/amcis2007/339/

Ellis, K. (2010). Be Who You Want to Be: The Philosophy of Facebook and the Construction of Identity. Screen Education, 58, 36-41.

Furht, B. (2010). Handbook of Social Network Technologies and Applications. New York: Springer.

Gilpin, D. R. (2011). Working the Twittersphere: Microblogging as professional identity construction. In Z. Papacharissi, A Networked Self (pp. 232-251). New York: Routledge.

Golbeck, J. (2005). Computing and Applying Trust in Web-based Social Networks. College Park: University of Maryland.

Hampton, K., Goulet, L. S., Rainie, L., \& Purcell, K. (2011). Social networking sites and our lives. Washington: Pew Research Center's Internet \& American Life Project.

Herring, S. C. (2010). Web Content Analysis: Expanding the Paradigm. In: Hunsinger, L. Klastrup, \& M. Allen (Eds.), International Handbook of Internet Research (pp. 233-251). Dortberch Heidelberg London New York: Springer.

Herring, S. C., Kouper, I., Paolillo, J. C., Scheidt, L. A., Tyworth, M., Welsch, P., Wright, E., \& Yu, N. (2005). Conversations in the blogosphere: An analysis "from the bottom up." Proceedings of the Thirty-Eighth Hawai'i International Conference on System Sciences (HICSS-38). Los Alamitos: IEEE Press. Retrieved from http://ella.slis.indiana.edu/ herring/blogconv.pdf.

Herring, S. C., Scheidt, L. A., Bonus, S., \& Wright, E. (2004). Bridging the gap: A genre analysis of weblogs. Proceedings of the 37th Hawai'i International Conference on System Sciences (HICSS-37). Los Alamitos: IEEE Computer Society Press. Retrieved from http:/ / ella.slis.indiana.edu/ herring/herring.scheidt.2004.pdf.

Herring, S. C., Scheidt, L. A., Bonus, S., \& Wright, E. (2005). Weblogs as a bridging genre. Information, Technology \& People, 18(2), 142-171. Retrieved from http:/ / ella.slis.indiana.edu/ herring/itp.pdf.

Herring, S. C., Scheidt, L. A., Kouper, I., \& Wright, E. (2006). A longitudinal content analysis of weblogs: 2003-2004. In M. Tremayne (Ed.), Blogging, Citizenship, and the Future of Media (pp. 3-20). London: Routledge. Preprint. Retrieved from: http:/ / ella.slis.indiana.edu/ herring/tremayne.pdf.

Johnson, H., Lavesson, N., Zhao, H., \& Wu, S. F. (2011). On the Concept of Trust in Online Social Networks. In: N. Blefari-Melazzi, G. Bianchi, \& L. Salgarelli, Trustworthy Internet (pp. 143-159). Milan: Springer.

Lewis, D., \& Weigert, A. (1985). Trust as a Social Reality. Social Forces, 63(4), 976-985.

Mehdizadeh, S. (2010). Self-Presentation 2.0: Narcissism and Self-Esteem on Facebook. Cyberpsychology, Behavior, and Social Networking, 13(4), 357-364.

Smith, M. (2009). A New Model for Trust and Reputation: Learning about competence and commitment. College Park: University of Maryland.

Tran, L. (2009). Trust in an online hospitality network: An interpretive study of The CouchSurfing Project. Recuperado el 7 de April de 2012, de Høgskolen i Oslo. Avdeling for journalistikk, bibliotek- og informasjonsvitenskap. Retrieved from: http://hdl.handle.net/10642/877.

Trepte, S., \& Reinecke, L. (2011). Privacy Online: Perspectives on Privacy and Self-disclosure in the Social Web. Berlin: Springer-Verlag.

World Values Survey Association. (2005). World Values Survey 2005 Official Data File. Version v20090901. Retrieved from http:/ / www.worldvaluessurvey.org.

Zhang, J. (2004). Familiarity and trust: Measuring familiarity with a web site. Proceedings of the 2nd Annual Conference on Privacy, Trust and Security (pp. 23-28). Fredericton, Canada: PST 2004.

Zhao, S., Grasmuck, S., \& Martin, J. (2008). Identity construction on Facebook: Digital empowerment in anchored relationships. Computers in Human Behavior, 24(5), 1816-1836.

Zucker, L. (1986). Production of Trust. Institutional Sources of Economic Structure. In: B. S. (eds), Research in Organizational behaviour, 8, 53-111. London: JAI Press. 